\title{
Ensino e aprendizagem da prática profissional: o caso dos supervisores de estágio do curso de graduação em Terapia Ocupacional da UFSCar
}

\section{Professional practice teaching-learning process: the supervisors' case from UFSCar Occupational Therapy undergraduate course}

\author{
Paula Signe Beutel $^{1}$, Gerusa Ferreira Lourenço² ${ }^{2}$, Taís Quevedo Marcolino ${ }^{3}$
}

http://dx.doi.org/10.11606/issn.2238-6149.v28i1p27-35

Beutel PS, Lourenço GF, Marcolino TQ. Ensino e aprendizagem da prática profissional: o caso dos supervisores de estágio do curso de graduação em Terapia Ocupacional da UFSCar. Rev Ter Ocup Univ São Paulo. 2017 jan.-abr.;28(1):27-35.

RESUMO: Considerando que o supervisor de estágio assume papel fundamental na formação prática profissional, este artigo busca elucidar aspectos do processo de ensino da prática em terapia ocupacional no período do estágio supervisionado bem como dificuldades e necessidades encontradas pelos profissionais clínicos para desempenharem o papel de supervisor. Trata-se de uma pesquisa qualitativa, realizada pela aplicação de questionário online aos supervisores de estágios do curso de graduação de Terapia Ocupacional da Universidade Federal de São Carlos e análise temática dos dados. Os resultados indicam que as sete supervisoras participantes apresentaram questões similares relativas à preparação para o papel de supervisor; às suas demandas e dificuldades; e à avaliação da relação universidade-serviço. Esperase que este trabalho contribua para a discussão sobre o processo de ensino-aprendizagem da prática profissional na graduação bem como para oferecer subsídios para reflexões sobre a capacitação de supervisores em terapia ocupacional.

DESCRITORES: Terapia ocupacional/educação; Desenvolvimento de pessoal; Prática profissional; Educação; Ensino/recursos humanos; Estágios; Capacitação de recursos humanos em saúde.
Beutel PS, Lourenço GF, Marcolino TQ. Professional practice teaching-learning process: the supervisors' case from UFSCar Occupational Therapy undergraduate course. Rev Ter Ocup Univ São Paulo. 2017 Jan.-Apr.;28(1):27-35.

ABSTRACT: The supervisor assumes a key role in professional training. This article elucidates aspects of fieldwork educational process in occupational therapy and the difficulties and needs encountered by clinicians to perform the supervisory role. We worked in a qualitative research, carried out applying an online questionnaire to fieldwork supervisors of an undergraduate Occupational Therapy course, at Federal University of Sao Carlos, with data analysed by thematic analysis. The results indicate that the seven research subject had similar issues related to the preparation for the role of supervisor; their demands and difficulties on supervision processes; and the relationship between University and service. It is expected that this work contribute to the discussion of professional practice teaching-learning process for undergraduate students as well to offer subsidies for reflections on occupational therapy supervisors training.

KEYWORDS: Occupational therapy/education; Staff development; Professional practice; Education; Teaching/ manpower; Internships; Health human resource training.

Este manuscrito é resultado do Trabalho de Conclusão do Curso de Graduação em Terapia Ocupacional da Universidade Federal de São Carlos da primeira autora, sob orientação da terceira autora.

1. Terapeuta Ocupacional. E-mail: paulasbeutel@gmail.com

2. Professora Adjunta do Departamento de Terapia ocupacional e do Programa de Pós-Graduação em Educação Especial da Universidade Federal de São Carlos, Brasil. E-mail: gerusalourenco@gmail.com

3. Professora Adjunta do Departamento de Terapia ocupacional e do Programa de Pós-Graduação em Terapia Ocupacional da Universidade Federal de São Carlos, Brasil. E-mail: taisquevedo@gmail.com

Endereço para correspondência: Taís Quevedo Marcolino. Departamento de Terapia Ocupacional - UFSCar. Rod. Washington Luis, km 235. São Carlos, SP, Brasil. CEP: 13565-905. E-mail: taisquevedo@gmail.com 


\section{INTRODUÇÃO}

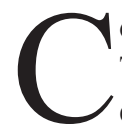
onsiderando que a prática profissional em Terapia Ocupacional encontra-se imersa em situações complexas, singulares e com conflito de valores ${ }^{1,2}$, a aprendizagem da prática na formação inicial, em uma perspectiva que valorize o conhecimento produzido na prática (não somente como aplicação de teorias), demanda a aproximação do estudante à realidade e complexidade dos serviços, mediado por terapeutas ocupacionais, como supervisores ${ }^{3-7}$. Esse processo depende da construção da relação e do diálogo entre supervisor e estudante, da compreensão do estilo de aprendizagem do aluno, e da construção de uma relação "de confiança e dependência" (p.80) ${ }^{1}$, que promoverá a autonomia do estudante, além da oferta de suporte subjetivo e emocional, e de feedback ${ }^{4,8-10}$.

Muitos trabalhos na área abordam a questão do ensino-aprendizagem no estágio em uma tentativa de avaliação, revisão e busca de novas estratégias para melhor adequá-lo às exigências sociais ${ }^{11,12}$. A supervisão aqui tratada se constitui como o espaço de diálogo entre o aluno e o terapeuta ocupacional do serviço no papel de seu supervisor, com o intuito de mediar o processo de ensino-aprendizado da prática profissional. Os desenhos estabelecidos para esse diálogo são diversos, porém com a convergência de contemplarem, pelo acompanhamento das ações realizadas pelos alunos, o ensino do raciocínio profissional e das habilidades necessárias para o cuidado em terapia ocupacional ressonantes com o campo específico e com o funcionamento institucional, em um processo de avaliação formativa ${ }^{2}$. Portanto, é apresentada como elemento condutor desse processo, circunscrita às características do serviço e ao campo de atuação, além de características particulares do supervisor, como seu estilo, suas habilidades e competências, e a constante atualização teórica e prática, domínio teórico-clínico, e habilidades didáticas ${ }^{8-10}$.

Assim, grupos colaborativos de aprendizagem; aprendizagem com os pares/colegas; aprendizagem baseada em problemas; metodologia de estudo de caso; seminários de raciocínio clínico, discussões de caso coletivas permitindo um diálogo multidisciplinar, são as estratégias encontradas na literatura ${ }^{4,11,13-15}$. Tais estratégias valorizam a colaboração entre os estudantes como fator importante para a aquisição de autonomia profissional e da aprendizagem do trabalho interprofissional, apontada como um dos desafios para uma formação voltada para as necessidades sociais contemporâneas ${ }^{15,16}$.

No Brasil, pesquisas sobre o ensino da clínica de terapia ocupacional no Brasil são escassas. Desde a década de 1990, há estudos que buscam discutir metodologias de ensino no estágio supervisionado ${ }^{17,18}$ e relatos de experiências de estagiários e supervisores durante o processo de aprendizagem ${ }^{19,20}$. Esses trabalhos indicam benefícios da supervisão para os estudantes em formação inicial em terapia ocupacional: a) maior segurança para atuação em determinada área; b) incentivo ao estudo teórico, necessário à fundamentação das práticas experimentadas no estágio; c) expressão de dúvidas, ansiedades e insegurança no transcorrer dessa etapa.

Vasconcellos e Almeida ${ }^{6}$, ao investigar egressos do curso de graduação em Terapia Ocupacional da Universidade de São Paulo, Campus de São Paulo, formados entre 2007 e 2010, pontuam que o estágio profissionalizante foi considerado um espaço favorável para ampliação de conhecimentos, habilidades e práticas para atuação profissional, mas indicam a necessidade de ajustes quanto à ampliação de referenciais, cenários e carga horária destinada a supervisão.

Embora se reconheça a importância do supervisor no processo de aprendizagem da prática profissional, vislumbramos a necessidade de maior sistematização dessa temática, em especial na realidade brasileira, inclusive face às novas propostas de ensino dos profissionais da área da saúde no Brasil. Assim, esse trabalho busca elucidar aspectos do ensino da prática profissional em Terapia Ocupacional no período do estágio supervisionado, na perspectiva dos supervisores, indicando potências, dificuldades e necessidades encontradas pelos profissionais nesse processo.

\section{METODOLOGIA}

Trata-se de uma pesquisa qualitativa, realizada com as supervisoras do curso de graduação de Terapia Ocupacional da Universidade Federal de São Carlos (UFSCar).

Em 2008, o curso de Terapia Ocupacional da UFSCar implantou um novo projeto político-pedagógico, sustentandose em metodologias ativas de aprendizagem, principalmente na Aprendizagem Baseada em Problemas (ABP) e na Problematização $0^{21}$. O currículo organizava-se em 5 unidades educacionais - de prática supervisionada; de referenciais teóricos e metodológicos; de pesquisa; de recursos e

Em 2015 foi aprovado um novo projeto político-pedagógico, entretanto, a estrutura de práticas e estágios supervisionados foi a que menos sofreu alteração $0^{22}$. 
Beutel PS, et al. Ensino e aprendizagem da prática profissional. Rev Ter Ocup Univ São Paulo. 2017 jan./abr.;28(1):27-35.

atividades; de consultoria - que se desenvolviam em diferentes graus de complexidade ao longo dos 5 anos de curso*.

A Unidade Educacional de Prática Supervisionada em Terapia Ocupacional sustenta-se na vivência dos estudantes em contextos reais e o conhecimento é construído por meio conteúdos (cognitivo, afetivo, psicomotor) emergentes das questões da prática ${ }^{22}$.

No quarto e quinto anos, os estudantes desenvolvem estágios profissionais em equipamentos de saúde, educação e do campo social, tanto sob supervisão de docentes da universidade como de terapeutas ocupacionais de serviços públicos ou privados, na função de supervisores de estágio ${ }^{22}$.

A coleta de dados foi realizada em novembro de 2014, por meio um questionário em ambiente virtual enviado a dezoito supervisores de estágio, sendo nove de instituições externas à UFSCar e nove docentes da UFSCar, responsáveis pelas disciplinas de estágio supervisionado. $\mathrm{O}$ questionário continha dados de identificação e formação profissional, e questões discursivas relativas a (a) atuação profissional (campo de atuação, tempo de experiência e características da população-alvo do campo), (b) processo de ensino e aprendizagem da prática (tempo de experiência como supervisor, principais demandas e dificuldades, compreensão sobre tal processo), (c) processo de supervisão (frequência da supervisão, principais características, planejamento, feedback), e (d) aspectos específicos da instituição e do campo de prática (avaliação da duração do estágio; benefícios da supervisão).

O projeto foi aprovado pelo Comitê de Ética da Universidade Federal de São Carlos sob parecer n ${ }^{\circ}$ 902.435.

Os dados de caracterização das supervisoras foram analisados por estatística simples descritiva e os dados qualitativos, tematicamente, para cada questão, considerando-se as três etapas de análise ${ }^{23}$ : etapa preliminar (escolha dos documentos a serem analisados); exploração do material (categorização das expressões ou palavras significativas no material de modo a organizá-lo); e tratamento dos resultados obtidos (interpretação que coloque em evidência os tópicos que mais apareceram ou menos) interpretados à luz dos objetivos do projeto.

\section{RESULTADOS}

\section{Caracterização das supervisoras}

Responderam ao questionário 7 supervisoras, de um total de 18 convidados, sendo 3 do quadro docente da UFSCar e 4 profissionais dos serviços que oferecem estágio, externos à Universidade. Embora o número de respondentes tenha sido pequeno (aproximadamente $40 \%$ ), a característica das supervisoras em relação à qualidade da formação, grande experiência na área e tempo na atividade de supervisão de estágio fez com que avaliássemos os resultados à luz das pesquisas com profissionais experientes ${ }^{24}$, valorizando o conhecimento prático em contexto.

O tempo médio de conclusão da formação graduada foi de 19 anos, sendo o máximo 39 anos e o mínimo 3 anos. Em relação à formação pós-graduada: seis possuíam cursos de especialização ou de formação clínica (Especialização em Tecnologia Assistiva e Terapia da Mão, Conceito Bobath, Saúde Mental pelo Centro de Especialidades em Terapia Ocupacional/CETO, Aperfeiçoamento em Psicanálise, Especialização em Saúde da Família); quatro possuíam Mestrado (áreas: Terapia Ocupacional, Educação, Terapia Ocupacional/ Acessibilidade); e dois haviam concluído o Doutorado (Bioengenharia e Educação). O tempo médio do exercício da função de supervisora de estágio foi de 7 anos e meio, sendo o máximo 30 anos e o mínimo 3 meses.

\section{Organização e características dos estágios e da supervisão}

O Quadro 1 apresenta as principais características dos estágios.

Os estágios oferecidos apresentaram duração média de quatro meses, variando de 16 a 20 horas semanais, destinadas à prática, supervisão, escrita de relatórios e estudos direcionados como leitura de material teórico.

A supervisão acontecia semanalmente em todas as instituições, e eram destinadas somente a estudantes de terapia ocupacional. Duas respondentes informaram que a supervisão era individual (Supervisora D e G), e em outras três instituições, ela ocorria tanto em modo individual como grupal ressaltando-se que a supervisão individual dava-se quando a supervisora desejava fazer observações pontuais e/ou relacionadas a questões pessoais do aluno, sendo uma das ações ou desenhos possíveis contemplados no processo de supervisão (Supervisoras A, B e F).

Em duas instituições (A e B) aconteciam reuniões interdisciplinares, das quais participavam vários profissionais da equipe e/ou estagiários de diferentes áreas, e a Supervisora $\mathrm{C}$ informou que faz supervisões com estagiários de terapia ocupacional de diferentes universidades que compõem o estágio. 
Beutel PS, et al. Ensino e aprendizagem da prática profissional. Rev Ter Ocup Univ São Paulo. 2017 jan./abr.;28(1):27-35.

Quadro 1 - Resultados da Análise Temática por Categoria e Participante

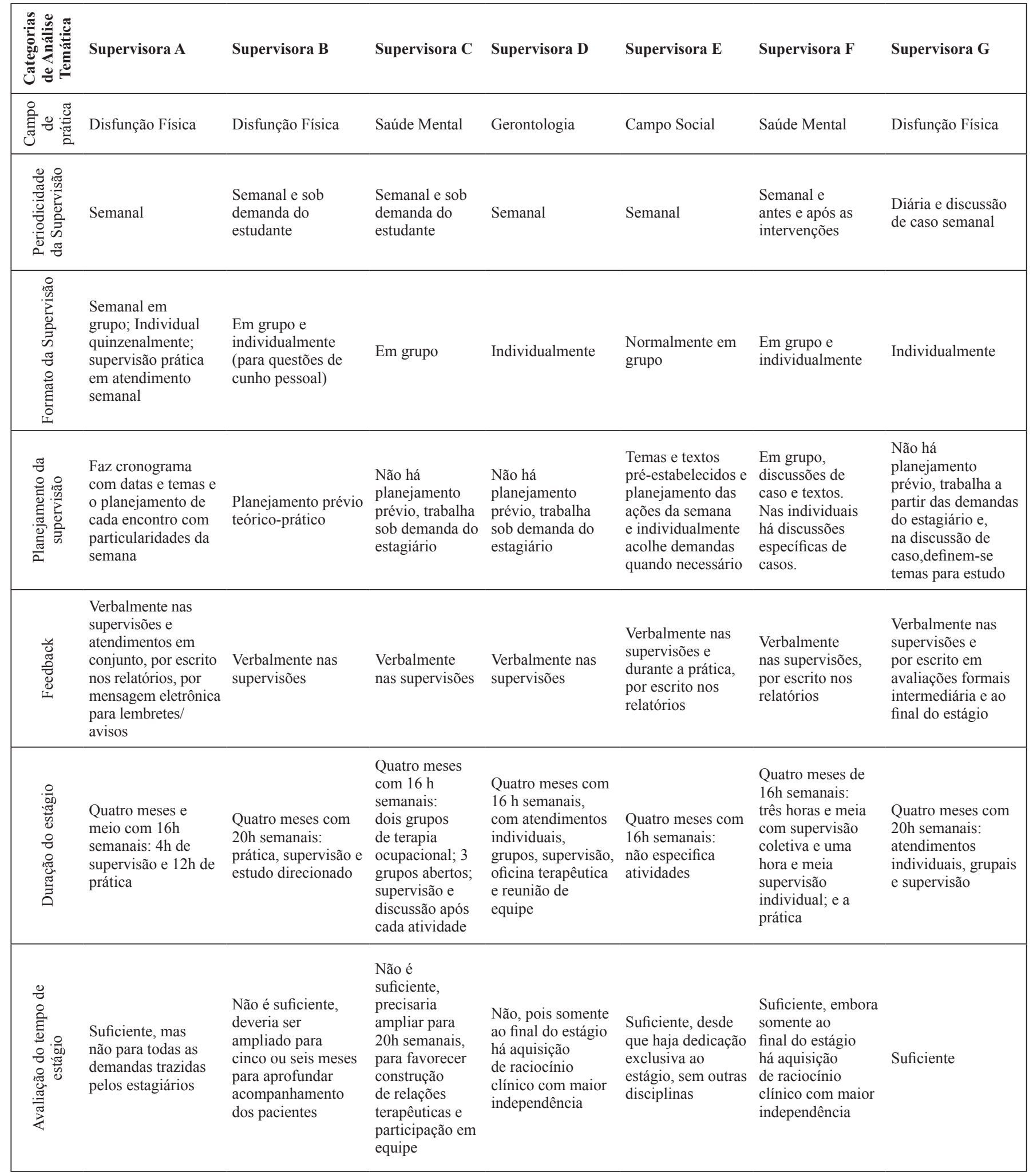


Beutel PS, et al. Ensino e aprendizagem da prática profissional. Rev Ter Ocup Univ São Paulo. 2017 jan./abr.;28(1):27-35.

Das sete supervisoras que responderam ao questionário, cinco planejavam a supervisão previamente, levando em consideração: questões teóricas a serem discutidas; material textual indicado para estudo; leitura de relatórios dos alunos para posterior devolutiva; e levantamento de questões relacionadas às demandas que os estagiários trazem. As duas supervisoras que referiram não planejar a supervisão apontavam-na como um espaço aberto para os estagiários pudessem trazer dúvidas e questionamentos acerca da experiência vivida.

Todos referiram que ofereciam materiais de estudo específicos à temática e às demandas que os estagiários traziam, assim como incentivam os estudantes a buscaremnas ativamente.

Os feedbacks eram oferecidos constantemente aos estudantes, não somente durante as avaliações formais, mas, verbalmente, durante as supervisões e atendimentos, ao explicar determinado manuseio ou procedimento, ou por escrito, por meio dos relatórios. As supervisoras A, C, $\mathrm{D}$ e $\mathrm{E}$ frisaram valorizar os aspectos positivos dos alunos e, quando havia assuntos delicados ou problemas mais graves, eram realizadas conversas individuais.

Em relação ao tempo de duração dos estágios em quatro meses (que variou de dezesseis a vinte horas semanais) quatro supervisoras (A, E, F e G) julgaram esse tempo suficiente para conhecer o campo de atuação e suas demandas, mas indicaram ressalvas quanto ao conhecimento prévio que os estudantes traziam sobre a área que, por vezes, apresentava-se frágil para a atuação, o que demandava o estudo teórico, ocupando o tempo da prática.

As supervisoras que acreditavam que o tempo de duração deveria ser maior, pelo menos de seis meses, discutiram que tanto o estudante (por seu ganho de maturidade no processo), como os usuários do serviço (por receberem um tratamento mais completo, com expansão do tempo de acompanhamento pelo estagiário) se beneficiariam.

"[...] quando o aluno começa a ficar mais à vontade no contato com o paciente, ou começa a fazer um raciocínio clínico com um pouco de independência (...) já está na hora de finalizar o estágio”. (Supervisora D)

"[...] seria melhor aproveitado para aprofundar mais o acompanhamento do paciente com estabelecimento de um plano de tratamento mais completo". (Supervisora B)

A supervisora E foi mais além, trazendo a questão do estágio profissionalizante ser realizado concomitantemente com disciplinas da faculdade e/ ou o trabalho de conclusão de curso, atrapalhando o desempenho do estudante, que precisa se dedicar a outras atividades que não somente ao estágio.

\section{Preparação para o papel de supervisor}

Das sete supervisoras participantes, seis se sentiam preparadas para exercer a função de supervisão, sendo que a única que respondeu não se sentir preparada, afirmou estar sempre buscando se capacitar e referindo uma constante busca de aprimoramento.

"[...] é importante eu estar segura de minha atuação enquanto terapeuta ocupacional e poder ter um embasamento clínico e metodológico que permita sua aprendizagem no serviço". (Supervisora G)

As que se sentiam preparadas para exercer a função elencaram pontos importantes que o supervisor deve desenvolver para garantir que os estudantes tenham um ensino de qualidade: embasamento científico; experiência no campo de atuação; didática; e, principalmente, domínio teórico-clínico.

Além de suporte técnico, as supervisoras também referiram oferecer suporte emocional, acolhendo incertezas e inseguranças do estagiário, de modo que ele possa tanto superar os desafios com que se deparam nos diferentes serviços, como colocando-se como modelo de postura profissional, auxiliando sua formação pessoal, teórica, prática e ética.

"Sem dúvida é incentivada também a pesquisa de questões relacionadas/suscitadas pela experiência do estágio, $e$ entendo também que esse espaço formativo deve se configurar como um espaço reflexivo, no qual o aluno possa ter suporte para lidar com as angústias provocadas pelo encontro com o sofrimento dos pacientes, com o não saber como lidar com diferentes questões". (Supervisora E)

As supervisoras trouxeram que o contato com os estagiários as levam a estar sempre se atualizando em relação a temas e assuntos pertinentes à prática profissional para conseguir dar um suporte maior e melhor para o estagiário, avaliando constantemente sua prática profissional. Referiram que a experiência que possuem na área as ajudam a entender melhor as dúvidas e anseios dos estudantes, conseguindo transmitir muito de seu conhecimento por meio de ações e exemplos práticos, que só a experiência proporciona.

“[...] o processo de ensino e aprendizagem da prática profissional traz um constante movimento de renovação e deve provocar no supervisor também questionamentos sobre sua prática, seus preceitos". (Supervisora C) 
Beutel PS, et al. Ensino e aprendizagem da prática profissional. Rev Ter Ocup Univ São Paulo. 2017 jan./abr.;28(1):27-35.

"Desmistifica algumas verdades, eles nos questionam inclusive condutas terapêticas que tomamos $e$ ai precisamos nos munir de muitas respostas". (Supervisora F)

\section{Demandas e dificuldades das supervisoras}

As demandas e dificuldades trazidas pelas supervisoras abarcaram desde a avaliação e escolha dos pacientes que os estagiários irão acompanhar a questões de ordem teórica. A formação prévia ao campo foi indicada como problemática, levando a supervisora a dedicar o tempo da supervisão - que poderia ser usado para discutir a prática - para preencher lacunas, levando-as a preparar aulas e organizar materiais de estudo para complementação teórica - o que relataram tensionar a qualidade do estágio em relação ao seu tempo de duração.

Em relação ao ensino da prática profissional e aos aspectos essenciais para um bom processo de supervisão, as supervisoras não referiram dificuldades específicas, mas refletiram sobre sua atuação nas dificuldades vividas pelos estagiários (angústias; dúvidas; dificuldades com o campo de atuação, quando não é seu preferido) de modo a ajudá-los a encontrarem uma imagem do profissional que gostariam de ser, ressaltando a necessidade de se colocarem disponíveis para esse trabalho.

"[...] nesse processo de formação profissional que na maior parte das vezes não é fácil, pois envolve muitas questões: afinidade com a área, confronto com valores morais e pessoais, crises de identidade profissional etc". (Supervisora E)

"Motivação da parte do aluno em aprender, mesmo que ainda não tenha identificado a área de atuação que 'deseja'”. (Supervisora B)

"Eu penso que o supervisor tem como tarefa olhar de fora e ver onde está a dificuldade, ajudar o aluno a pensar sobre o que está fazendo". (Supervisora D)

\section{Relação universidade-serviço}

Das sete supervisoras, três eram docentes da UFSCar e quatro, profissionais de instituições de saúde. Assim, nesse item, consideramos apenas as respostas provenientes dos supervisores externos, uma vez que as três docentes indicaram que a universidade é a própria instituição gestora do serviço.

De modo geral, avaliaram a relação entre as instituições como sendo boa ou muito boa, mas enfatizaram a necessidade de aproximação. Uma supervisora pontuou que a relação é muito boa e, por ser de longa data, está consolidada.

\section{“[..]jábastante antiga e com sólida tradição semprefoimuito boa e aberta às composições das diferentes peculiaridades tanto institucionais, como do acompanhamento das necessidades de cada aluno". (Supervisora C)}

Outras três (supervisoras B, D e G), apesar de consideraram boa a relação com a universidade, acreditam que ela deveria ser mais próxima, de modo a haver um retorno constante da universidade sobre o processo de estágio, assim como aumentar a integração das atividades de estágio e acadêmicas, como visitas institucionais em outros momentos do curso - o que talvez pudesse ajudar a suprir as lacunas de conteúdo apresentada pelos estudantes.

\footnotetext{
"As visitas nas instituições também são uma maneira importante de manter este contato entre a instituição, a universidade e os alunos e deveriam ocorrer com maior frequência". (Supervisora G)

"Acredito que uma maior aproximação com os supervisores e docentes da universidade seria importante para uma maior integração das atividades desenvolvidas no estágio com as atividades acadêmicas". (Supervisora B)
}

\section{DISCUSSÃO}

Todas as supervisoras que participaram desta pesquisa tinham formação pós-graduada, experiência profissional acima de dois anos, e a maior parte delas com experiência em supervisão de estágios, o que nos indicou ser um grupo com bom domínio na temática da pesquisa. Em nossa pesquisa obtivemos resultados similares a outros trabalhos ${ }^{4,8,10}$, indicando que para exercer a função de supervisor é necessário embasamento científico; experiência no campo de atuação; habilidades didáticas; e, principalmente, domínio teórico-clínico.

A supervisão em grupo foi o formato mais comum dos estágios, sendo considerada uma estratégia positiva na formação profissional tanto para os estudantes, ao compartilhar suas experiências e construir juntos o processo de aprendizagem, como para o supervisor, que 
Beutel PS, et al. Ensino e aprendizagem da prática profissional. Rev Ter Ocup Univ São Paulo. 2017 jan./abr.;28(1):27-35.

se coloca direcionando a aprendizagem, sem conduzila por completo, diminuindo suas responsabilidades e aumentando a autonomia dos estagiários ${ }^{14}$.

Entretanto, encontramos poucas experiências interprofissionais, circunscritas a discussões de casos em toda a equipe do serviço, não se caracterizando, portanto, como uma estratégia formativa assumida pelos serviços. Tal aspecto vem a indicar uma importante fragilidade na formação, que não vai ao encontro das diretrizes curriculares $^{25}$, nem de uma formação voltada para as necessidades sociais contemporâneas ${ }^{16}$.

As supervisoras participantes também indicaram a grande demanda de oferta de suporte emocional e subjetivo $^{8}$. Por ser uma fase de transição do estudante para a vida profissional, essa característica nos sugere que a supervisão é, de fato, uma estratégia formativa importante para a iniciação profissional, no sentido de ser sensível aos aspectos subjetivos do estudante, para conduzi-lo à construção de sentidos sobre ser terapeuta ocupacional, ser profissional e lidar com os desafios reais do trabalho.

Uma estratégia referida para lidar com essas questões foi a supervisão individual, solicitada, muitas vezes, pelo estudante e mais direcionada a questões pontuais, angústias que ele não pode ou não quer compartilhar com os demais. Embora alguns autores ${ }^{13}$ avaliem negativamente a supervisão individual, referindo que o estudante pode criar uma relação de dependência com o supervisor, o que não condiz com a realidade profissional que ele irá se deparar futuramente, essa estratégia também pode ser vista em seus aspectos positivos, ao propiciar o estreitamento da confiança que se estabelece entre supervisor e estudante.

Nesse ponto, Schön ${ }^{1}$, ao propor um arcabouço teórico para discutir a aprendizagem da prática profissional em uma perspectiva que valoriza o conhecimento produzido na prática (não somente como aplicação de teorias), destaca a importância da relação e do diálogo entre o instrutor/ supervisor e estudante. Esse autor destaca o dilema da dependência vivido pelo supervisor e pelo estudante, pois o supervisor pede ao estudante que dê um salto no escuro, que acredite que irá aprender somente se começar a praticar, e que precisa confiar no supervisor como alguém que vai lhe ensinar. Assim, mesmo desejando a autonomia do estudante no futuro, o supervisor pede que o estudante suspenda sua desconfiança e que aceite entrar em uma relação "de confiança e dependência", mesmo que temporariamente (p.80) $)^{1}$, para assim ganhar sua autonomia.

Além disso, alguns supervisores referiram que também se veem sendo colocados no lugar de modelo a seguir, e percebem que os estudantes se espelham neles para conseguir criar/encontrar o seu próprio perfil profissional, que também pode ser um aspecto dessa relação de dependência e confiança temporária ${ }^{1}$, nas experimentações sobre o profissional que o estudante deseja, ou não, ser.

Esses aspectos mais sutis do processo de aprendizagem da prática não foram aprofundados em nossa pesquisa, assim, novas investigações se fazem necessárias no futuro de modo a tecer relações mais férteis e complexas a respeito das relações vistas como positivas para o desenvolvimento da experiência do estágio profissional.

Como visto em outros estudos ${ }^{5,8}$, o contato com os estagiários demanda constante atualização técnicocientífica. As supervisoras referiram constante avaliação, não somente de seus conhecimentos, mas da própria prática profissional, valorizando a experiência cotidiana para compreender mais adequadamente o que se mostra problemático para o estudante, assim como a facilidade de ensinar por meio de ações e exemplos práticos, que só a experiência proporciona. Schön ${ }^{1}$ ressalta justamente esse aspecto do ensino-aprendizagem da prática, na medida em que o conhecimento está internalizado em cada um, sem que se consiga explicar em palavras o que se sabe fazer, precisando muitas vezes, demonstrar o que se quer ensinar. Esse autor discorre sobre o demonstrar-imitar, pois há coisas que o instrutor terá que mostrar visualmente, para que o aluno o imite e a partir daí, crie seu próprio jeito de desempenhar a atividade, de realizar a ação.

Um dos principais desafios para a formação prática indicado pelos supervisores refere-se ao descompasso entre a formação teórica prévia e o que é demandado na prática, levando-os, algumas vezes, a utilizar parte da carga horária ao estudo teórico e técnico para um repertório inicial no sentido de nivelamento quanto a requisitos para demandas específicas do determinado serviço ou instituição.

Considerando o projeto pedagógico do curso e os conceitos de aprendizagem pela prática e aprendizagem significativa, reconhece-se que de fato essa é uma questão dilemática, constantemente referida em vários estudos ${ }^{5,7,9}$. A maior proximidade entre a universidade e o serviço foi apontada como necessidade para superação desses desajustes, a qual traria benefícios para ambos os lados.

Assim, a supervisão pode ser compreendida como um processo complexo, que demanda preparação acadêmica, didática e emocional, de modo a garantir qualidade e enriquecer a experiência do estágio. Os supervisores referiram que a atuação junto aos estudantes é um aspecto gratificante da função e traz reconhecimento, ao participar da formação de futuros terapeutas ocupacionais $^{2,7,11}$. 
Beutel PS, et al. Ensino e aprendizagem da prática profissional. Rev Ter Ocup Univ São Paulo. 2017 jan./abr.;28(1):27-35.

\section{CONSIDERAÇÕES FINAIS}

Esse estudo trouxe um panorama geral das demandas e dificuldades pela quais passam os supervisores, a relação que estabelecem com os estagiários e com a universidade.

Foi possível caracterizar os estágios no que se refere à instituição, ao período de desenvolvimento e às características da supervisão. Além disso, nosso estudo pode oferecer elementos para discutir, do ponto de vista dos supervisores, suas necessidades de formação, e as relações que se estabelecem entre a experiência profissional e os aspectos que circundam a aprendizagem da prática.

As limitações do estudo referem-se principalmente: a) à pequena amostra de sujeitos que participaram da pesquisa, favorecendo o levantamento dos temas mas não

\section{REFERÊNCIAS}

1. Schön D. Educando o profissional reflexivo. Porto Alegre: Artmed; 2000.

2. Marcolino TQ, Lourenço, GF, Reali AMMR. "This is my take-home for life!": professional practice learning on a community of practice. Interface (Botucatu). 2017;21(61):411-20. doi: 10.1590/1807-57622016.0099.

3. Chiang HA, Pang C-H, Li W-S, Shih Y-N, Su C-T. An investigation of the satisfaction and perception of fieldwork experiences among occupational therapy students. Hong Kong J Occup Ther. 2012;22:9-16. doi: 10.1016/j.hkjot.2012.04.001.

4. Koski KJ, Simon RL, Dooley NR. Valuable occupational therapy fieldwork educator behaviors. Work. 2013;44(3):30715. doi: 10.3233/WOR-121507.

5. Bell J, Cox D, Marcangelo C. Masters level occupational therapy students on placement: an exploration of perceptions and expectations. Br J Occup Ther. 2014;77(4):181-8. doi: 10.4276/030802214X13968769798755.

6. Vasconcellos KMA., Almeida MHM. Percepção de egressos sobre estágios de terapia ocupacional em geriatria e gerontologia. Rev Ter Ocup Univ São Paulo. 2013;24(1):4856. doi: 10.11606/issn.2238-6149.v24i1p48-56.

7. Naidoo D, Mot JW. Fieldwork practice for learning: lessons from occupational therapy students and their supervisors. Afr J Health Professions Educ. 2016;8(1):37-40. doi: 10.7196/AJHPE.2016.v8i1.536

8. Rodger S, Thomas Y, Greber C, Broadbridge J, Edwards A, Newton J, Lyons M. Attributes of excellence in practice seu aprofundamento nem possibilidades de generalização do conhecimento; b) à variabilidade de formas de ensino da prática profissional com as quais os cursos da área da Saúde, de modo geral, e os de Terapia Ocupacional, de modo específico, vem trabalhando, dadas as propostas de reestruturação do ensino superior na área da saúde no Brasil ${ }^{25}$, nas quais a função de quem ensina a prática profissional ganha diferentes sentidos e matizes.

Dado o desafio da formação da prática profissional para a realidade contemporânea em Terapia Ocupacional, levantamos a necessidade de um contínuo de estudos nessa temática, de modo a aprofundar os conhecimentos a respeito do processo de ensino-aprendizagem envolvido, dando voz a supervisores/preceptores e a estudantes, e às relações estabelecidas entre os serviços formadores e as propostas pedagógicas dos cursos.

educators: The perspectives of Australian occupational therapy students. Austr Occup Ther J. 2014;61:159-67. doi: 10.1111/1440-1630.12096.

9. Towns E, Ashby S. The influence of practice educators on occupational therapy students' understanding of the practical applications of theoretical knowledge: a phenomenological study into student experiences of practice education. Austr Occup Ther J. 2014;61:344-52. doi. 10.1111/14401630.12134 .

10. Barton R, Corban A, Herrli-Warner L, McClain E, Riehle D, Tinner E. Role strain in occupational therapy fieldwork educators. Work. 2013;44:317-28. doi: 10.3233/WOR121508

11. Flood B, Haslam L, Hocking C. Implementing a collaborative model of student supervision in New Zealand: enhancing therapist and students experiences. $\mathrm{N}$ Z J Occup Ther. 2010;57(1):22-6. Available from: http://search.informit.com. au/documentSummary; $\mathrm{dn}=013323556063633$;res=IELNZC

12. Dancza K, Warren A, Copley J, Rodger S, Moran M, McKay E, Taylor A. Learning experiences on role-emerging placements: An exploration from the students' perspective. Austr Occup Ther J. 2013;60:427-35. doi. 10.1111/14401630.12079 .

13. Provident IM, Colmer MA. Muscular dystrophy summer camp: a case study of a non-traditional level I fieldwork using a collaborative supervision model. Work. 2014;44:337-44. doi: 10.3233/WOR-121510. 
Beutel PS, et al. Ensino e aprendizagem da prática profissional. Rev Ter Ocup Univ São Paulo. 2017 jan./abr.;28(1):27-35.

14. Copley J, Nelson A. Practice educator perspectives of multiple mentoring in diverse clinical settings. $\mathrm{Br} \mathrm{J}$ Occup Ther. 2012;75(10):456-62. doi: 10.4276/030802212X13496921049662.

15. Perrone MB, Bianchini APML, Fidalgo TM, Silveira DX. O ensino de Terapia Ocupacional na clínica das dependências: Uma experiência na liga acadêmica de farmacodependências (PROAD - UNIFESP). Cad Ter Ocup UFSCar. 2014;22(Supl Esp.):119-24. Disponível em: http:// www.cadernosdeterapiaocupacional.ufscar.br/index.php/ cadernos/article/view/842/511.

16. Camargo M, Bellini MIB. The tripod guiding of attention in the national healthcare service (SUS) in Brazil: integrality, interdisciplinary an intersectoriality. Doc Pol Soc. 2014;2(13):1-21. Available from: http://repositorio.pucrs. br/dspace/bitstream/10923/8092/2/The_tripod_guiding _ of attention_in the national_healthcare service_SUS in Brazil_integrality_interdisciplinary_and_intersectoriality.pdf.

17. Hahn M. O processo de supervisão em psiquiatria do aluno ou profissional. Cad Ter Ocup UFSCar. 1990;1(1):50-65. Disponível em: http://www.cadernosdeterapiaocupacional. ufscar.br/index.php/cadernos/article/view/10/15.

18. Pfeifer LI. Práticas pedagógicas em Terapia Ocupacional: capacitando o aluno para atuar junto a crianças com atraso no desenvolvimento. Rev Ter Ocup Univ São Paulo. 1997;8(2-3):75-80.

Recebido em: 13.09.16

Aceito em: 17.03.17
19. Marcolino TQ. Convite para pintar. Cad Ter Ocup UFSCar. 2001;9(1):50-6. Disponível em: http://www. cadernosdeterapiaocupacional.ufscar.br/index.php/cadernos/ article/view/234.

20. Barata DA, Cocenas SA, Kebbe LM. Coordenação de grupos de terapia ocupacional em enfermaria psiquiatrica relato de supervisão realizada com uma estagiária. Cad Ter Ocup UFSCar. 2010;18(2):181-90. Disponível em: http:// www.cadernosdeterapiaocupacional.ufscar.br/index.php/ cadernos/article/view/353/284.

21. Della Barba PCS, Silva RF, Joaquim RHVT, Brito CMD. Formação inovadora na graduação em Terapia Ocupacional. Interface (Botucatu). 2012;16:829-42. doi: 10.1590/S141432832012000300019 .

22. Universidade Federal de São Carlos - UFSCar. Projeto Político Pedagógico do Curso de Terapia Ocupacional da UFSCar. São Carlos: Departamento de Terapia Ocupacional; 2015.

23. Minayo MCS. O desafio do conhecimento: pesquisa qualitativa em saúde. 12a ed. São Paulo: Hucitec; 2010.

24. Robertson D, Warrender F, Barnard S. The critical occupational therapy practitioner: How to define expertise? Austr Occup Ther J. 2015;62:68-71. doi: 10.1111/1440-1630.12157.

25. Brasil. Conselho Nacional de Educação. Resolução CNE/ CES 6/2002. Diário Oficial da União, Brasília, 4 mar. 2002. Seção 1, p.12. 